Ю. НУРГЕС

\title{
МОДЕЛЬ АВТОРЕГРЕССИИ ОДНОГО КЛАССА БИЛИНЕИНЫХ СИСТЕМ
}

O. NURGES. BILINEAARSETE SUSTEEMIDE KLASSI AUTOREGRESSIOONMUDEL

D. NURGES. AUTOREGRESSIVE MODEL OF OUTPUT BILINEAR SYSTEMS

\author{
(Представил Н. Алумяэ)
}

Введение. Рассмотрим многомерную билинейную систему

$$
\begin{gathered}
x(t+1)=A x(t)+\sum_{i=1}^{m} u_{i}(\mathrm{t}) D_{i} x(t)+B u(t) \\
y(t)=C x(t)
\end{gathered}
$$

где $x n$-вектор состояния, $u-m$-вектор управляющих воздействий, $u_{i}-i$-й компонент вектора $u, y-p$-вектор выходных переменных системы. Матрицы $A$ и $D_{i}, i=1, \ldots, m$ имеют размерность $n \times n$, а матрицы $B$ и $C-n \times m$ и $p \times n$ соответственно.

Для идентификации системы необходимы соотношения между управляющими воздействиями и выходными переменными, т. е. надо элиминировать состояние $x(t)$ из уравнений (1) и $(2)$. Ввиду билинейности относительно состояния вход-выход модели билинейных систем оказываются громоздкими $\left[{ }^{1,2}\right]$. Более приемлемые результаты получены для одномерных систем со специальной структурой матрицы $D(\operatorname{rank} D=1)\left[{ }^{3}\right]$.

В данной работе выводится вход-выход соотношение типа авторегрессии для систем, бнлинейных относительно выхода, т. е. для систем, удовлетворяющих требованиям

$$
R\left(D_{i}\right) \subset R(C), \quad i=1, \ldots, m,
$$

где $R\left(D_{i}\right)$ - векторное пространство, порожденное строками матрицы $D_{i}$.

Вывод уравнения авторегрессии. При ограничении (3) найдутся матрицы $E_{i}, i=1, \ldots, m$ размерности $n \times p$ такие, что

$$
D_{i}=E_{i} C \text {, }
$$

и мы можем переписать уравнение состояния (1) следующим образом:

$$
x(t+1)=A x(t)+\sum_{i=1}^{m} u_{i}(t) E_{i} y(t)+B u(t) .
$$

Рассмотрим итерацию уравнений состояния (4) и выхода (2)

$$
y(t)=C x(t),
$$




$$
\begin{aligned}
y(t+1)= & C A x(t)+C \sum_{i=1}^{m} u_{i}(t) E_{i} y(t)+C B u(t) \\
& \\
y(t+n-p)= & C A^{n-p} x(t)+\sum_{j=0}^{n-p-1} C A^{n-p-j-1} \sum_{i=1}^{m} u_{i}(t+j) E_{i} y(t+j)+ \\
& +\sum_{j=0}^{n-p-1} C A^{n-p-j-1} B u(t+j),
\end{aligned}
$$

которую можно переписать в матричной форме

$$
P(u) \bar{y}(t)=\mathrm{O} x(t)+R \bar{u}(t),
$$

где $[\bar{y}(t)]^{\mathrm{T}}=\left[y^{\mathrm{T}}(t), \ldots, y^{\mathrm{T}}(t+n-p)\right]$,

$$
[\bar{u}(t)]^{\mathrm{T}}=\left[u^{\mathrm{T}}(t), \ldots, u^{\mathrm{T}}(t+n-p)\right],
$$$$
\mathrm{O}=\left[\begin{array}{l}
C \\
C A \\
\vdots \\
C A^{n-p}
\end{array}\right]
$$$$
R=\left[\begin{array}{cccc}
0 & 0 & \ldots & 0 \\
C B & 0 & \ldots & 0 \\
C A B & C B & & \\
\vdots & & & \vdots \\
\vdots & & \ddots & \vdots \\
C A^{n-p-1} B & \ldots & C B & 0
\end{array}\right]
$$$$
P(u)=\left[\begin{array}{ccccc}
I_{p} & 0 & 0 & \ldots & 0 \\
S_{0}(t) & I_{p} & 0 & \ldots & 0 \\
S_{1}(t) & S_{0}(t+1) & I_{p} & & \\
\cdot & \vdots & \ddots & \ddots \\
\vdots & \vdots & & \ddots & \vdots \\
S_{n-p-1}(t) & S_{n-p-2}(t+1) & \ldots & & I_{p}
\end{array}\right]
$$

$I_{p}-$ единичная матрица порядка $p \times p, S_{j}(t)=-C A^{j} \sum_{i} u_{i}(t) E_{i}$.

Для наблюдаемой пары $(A, C) \operatorname{rank} \mathrm{O}=n$ и

$$
\mathrm{O}^{+}=\left(\mathrm{O}^{\mathrm{T}} \mathrm{O}\right)^{-1} \mathrm{O}^{\mathrm{T}} \text {. }
$$

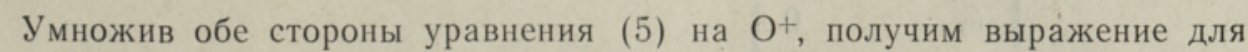
$x(t)$

$$
x(t)=\mathrm{O}^{+} P(u) \bar{y}(t)-\mathrm{O}^{+} \mathrm{R} \bar{u}(t) .
$$

Следующий шаг итерации дает 


$$
\begin{gathered}
y(t+n-p+1)=C A^{n-p+1} \mathrm{O}^{+}[P(u) \bar{y}(t)-R \bar{u}(t)]+ \\
+\sum_{j=0}^{n-p} C A^{n-p-j}\left[\sum_{i=1}^{m} u_{i}(t+j) E_{i} y(t+j)+B u(t+j)\right]
\end{gathered}
$$

или

$$
y(t+n-p+1)=\bar{P}(u) \bar{y}(t)+\bar{R} \bar{u}(t),
$$

где

$$
\begin{gathered}
\bar{P}(u)=C A^{n-p+1} \mathrm{O}^{+} P(u)+\left[C A^{n-p} \sum_{i} u_{i}(t) E_{i} \ldots: C \sum_{i} u_{i}(t+n-p) E_{i}\right], \\
\bar{R}=-C A^{n-p+1} \mathrm{O}^{+} R+\left[C A^{n-p} B: \ldots \vdots C B\right] .
\end{gathered}
$$

Уравнение (6) представляет собой авторегрессионную модель систем, билинейных относительно выхода. Число составляющих в модели (6) $N \leqslant(m+2)(n-p+1)+1$. Это гораздо меньше, чем в ссответствующей модели для систем, билинейных относительно состояния. Например, для $n=3, m=p=1 N=10$, в то время как модель авторегрессии систем, билинейных относительно состояния, включает 88 составляющих [ $\left.{ }^{1}\right]$.

Модель авторегрессии систем, билинейных относительно выхода, с одним входом и одним выходом. Пусть $m=p=1$. Ввиду наблюдаемости пары $(A, c)$ система $(1),(2)$ приводима к канонической форме

$$
\begin{aligned}
& A=\left[\begin{array}{ccc}
0 & 1 & I_{n-1} \\
\ldots & \ldots & \ldots \\
a_{1} & \ldots & a_{n}
\end{array}\right], \quad b=\left[\begin{array}{c}
b_{1} \\
\vdots \\
\vdots \\
b_{n}
\end{array}\right], \quad e=\left[\begin{array}{c}
e_{1} \\
\vdots \\
\vdots \\
e_{n}
\end{array}\right], \\
& c^{\mathrm{T}}=\left[\begin{array}{llll}
1 & 0 & \ldots & 0
\end{array}\right] .
\end{aligned}
$$

Тогда

$$
\begin{aligned}
\bar{P}(u) & =a^{\mathrm{T}}\left[I_{n}-P \cdot \operatorname{diag} \bar{u}(t)\right]+e^{\mathrm{T} J} \operatorname{diag} \bar{u}(t) . \\
\bar{R} & =-a^{\mathrm{T}} R+b^{\mathrm{T} J},
\end{aligned}
$$

где

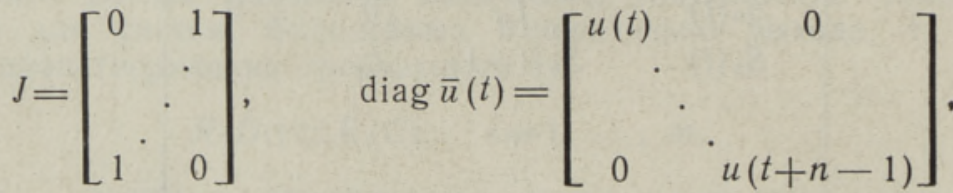

$$
\begin{aligned}
& P=\left[\begin{array}{cccc}
0 & & & \\
e_{1} & & & 0 \\
\vdots & \vdots & & \\
\vdots & \cdot & \cdot & \\
e_{n-1} & \ldots & e_{1} & 0
\end{array}\right] \text {, } \\
& R=\left[\begin{array}{cccc}
0 & & & \\
b_{1} & & & 0 \\
\cdot & \cdot & & \\
\cdot & \cdot & \cdot & \\
\dot{b}_{n-1} & \ldots & b_{1} & 0
\end{array}\right]
\end{aligned}
$$




$$
\begin{aligned}
y(t+n)= & a_{1} y(t)+a_{2} y(t+1)+\ldots+a_{n} y(t+n-1)+ \\
& +\left(e_{n}-a_{n} e_{n-1}-a_{n-1} e_{n-2}-\ldots-a_{2} e_{1}\right) u(t) y(t)+ \\
& +\left(e_{n-1}-a_{n} e_{n-2}-a_{n-1} e_{n-3}-\ldots-a_{3} e_{1}\right) u(t+1) y(t+1)+ \\
& +\ldots+\left(e_{2}-a_{n} e_{1}\right) u(t+n-2) y(t+n-2)+ \\
& +e_{1} u(t+n-1) y(t+n-1)+ \\
& +\left(b_{n}-a_{n} b_{n-1}-a_{n-1} b_{n-2}-\ldots-a_{2} b_{1}\right) u(t)+ \\
& +\left(b_{n-1}-a_{n} b_{n-2}-a_{n-1} b_{n-3}-\ldots-a_{3} b_{1}\right) u(t+1)+ \\
& +\ldots+\left(b_{2}-a_{n} b_{1}\right) u(t+n-2)+b_{1} u(t+n-1) .
\end{aligned}
$$

Заключение. Системы, билинейные относительно выхода, заслуживают тщательного изучения, так как по наблюдаемости и идентифицируемости они весьма близки к линейным. Их модель авторегрессии включает небольшое количество составляющих и тем самым подходит для решения задач идентификации и моделирования систем.

\section{Л И Т Е Р А Т У Р А}

1. Beghelli, S., Guidorzi, R. In: Identification and System Parameter Estimation, IV IFAC Symposium. Tbilisi, 1976, 360-370.

2. Inagaki, M. IEEE Trans, Automat. Contr., AC-27, № 4, 984-986 (1982).

3. Baheti, R. S., Mohler, R. R., Spang, H. A. IEEE Trans. Automat. Contr., AC-25, № 6, $1141-1146$ (1980).

Ннститут кибернетики Академии наук Эстонской ССР
Поступила в редакцию 15/II 1983 